\title{
Novel s-Triazinyl Schiff Base/Chalcone Congeners: Rational, Synthesis, Antimicrobial and Anti-TB Evaluation
}

\author{
Harshad P. Lakum, Dhruvin R. Shah, Kishor H. Chikhalia* \\ Department of Chemistry, School of Sciences, Gujarat University, \\ Ahmedabed - 380009, Gujarat, India \\ *Tel: +91-79-26300969/9427155529; Fax: +91-79-26308545 \\ *E-mail address: chikhalia_kh@yahoo.com
}

\begin{abstract}
The occurrence of Multi Drug Resistant (MDR) infectious microbial strains has been increased upto alarming level which affects the public health worldwide. To cure this problem, a library of $s$ triazinyl derivatives comprising schiff base or chalcone motif have been rationalized, synthesized and screened for their in vitro antibacterial activity against five bacterial strains (Staphylococcus aureus MTCC 96, Bacillus subtilis MTCC 441, Escherichia coli MTCC 739, Pseudomonas aeruginosa MTCC 741 and Klebsiella pneumoniae MTCC 109) and four fungal strains (Aspergillus niger MTCC 282, Aspergillus fumigates MTCC 343, Aspergillus clavatus MTCC 1323, Candida albicans MTCC 183 ) using broth dilution technique. All the newly synthesized scaffolds were further evaluated for their in vitro anti-TB efficacy against the tubercular strain (Mycobacterium tuberculosis H37Rv) using Lowenstein-Jensen MIC method. All the derivatives were well characterized by IR, ${ }^{1} \mathrm{H}$ NMR, ${ }^{13} \mathrm{C}$ NMR, elemental analysis as well as mass spectroscopy.
\end{abstract}

Keywords: Antimicrobial; Anti-TB; s-Triazine; Schiff Base; Chalcone

\section{INTRODUCTION}

Multidrug resistant strength of a variety of infectious microbial flora towards existing standard drugs has been increased in recent decade which is serious health problem worldwide. Antimicrobial resistant (AMR) is a phenomenon of infectious microbial flora to resist antimicrobial agents to which it was previously sensitive [1]. Various multidrug resistant microbes including bacteria, fungi, viruses, parasites etc. exhibit such type of resistant profile against traditional standard drugs including antibiotics, antifungals, antivirals, antimalarials etc. which results in failure or ineffectiveness of the standard treatment, so that the rate of spread of infectious disease also increases. The appearance of methicillin-resistant Staphylococcus aureus (MRSA), a multidrug resistant gram-positive bacterial strain, poses infectious disease. 
<smiles>CN(C)c1nc(N(C)C)nc(N(C)C)n1</smiles>

Altretamine

(Antineoplastic agent)<smiles>C1CN1c1nc(N2CC2)nc(N2CC2)n1</smiles>

Triethylenemelamine

(Chemotherapeutic agent)<smiles>CCNc1nc(Cl)nc(NC(C)C)n1</smiles>

Atrazine

(Herbicide)

Figure 1. s-Triazine based pharmaceutical agents.<smiles>O=C(O)COC(=O)O</smiles><smiles>N#Cc1ccc(I)cc1</smiles><smiles>CNc1nc(Cl)nc(Cl)n1</smiles><smiles>COC(=O)OCCOCCO</smiles><smiles>N#Cc1ccc(Nc2nc(Cl)nc(NC3CC3)n2)cc1</smiles>

2

3<smiles>N#Cc1ccc(N)cc1</smiles><smiles>Nc1nc(NC2CC2)nc(Oc2ccc(C=O)cc2)n1</smiles>

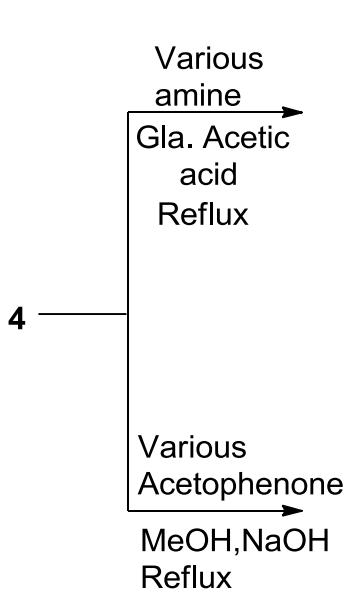<smiles>[R]N=Cc1ccc(Oc2nc(Nc3ccc(C#N)cc3)nc(NC3CC3)n2)cc1</smiles><smiles>[R]C(=O)/C=C/c1ccc(Oc2nc(Nc3ccc(C#N)cc3)nc(NC3CC3)n2)cc1</smiles>

$6 a-j$

Scheme 1. Synthetic pathway for the preparation final analogues 5a-j and 6a-j.

In the current period, some tubercular strains of Mycobacterium tuberculosis cause MDR-Tuberculosis (TB) and extensively drug-resistant XDR-TB which generally affects the lungs [2]. The current figures of World Health Organization (WHO) for the year 2012 showed 8.6 million people suffered with TB and 1.3 million died from TB. An estimated 530 
000 children became ill with TB and 74000 HIV-negative children died of TB [3]. In context of the above discussion, the cost of these troubles highlights the urgent need to develop new medicinal agents which have relatively higher efficiency to sustain a pool of new bioactive scaffolds.

In context of the above discussion, the cost of these troubles indicate the urgent need to develop new medicinal agents having relatively higher potency with reference to traditional drugs to sustain a pool of novel antimicrobial agents. Hence, rational and synthesis of novel bioactive agents likely to be unaffected by existing resistance mechanisms is an area of vast implication for medicinal chemists showed the urgent need to combat such impasse. The rationalization of novel bioactive agents with more selectivity and little toxicity persist an area of intensive research in synthetic medicinal chemistry.

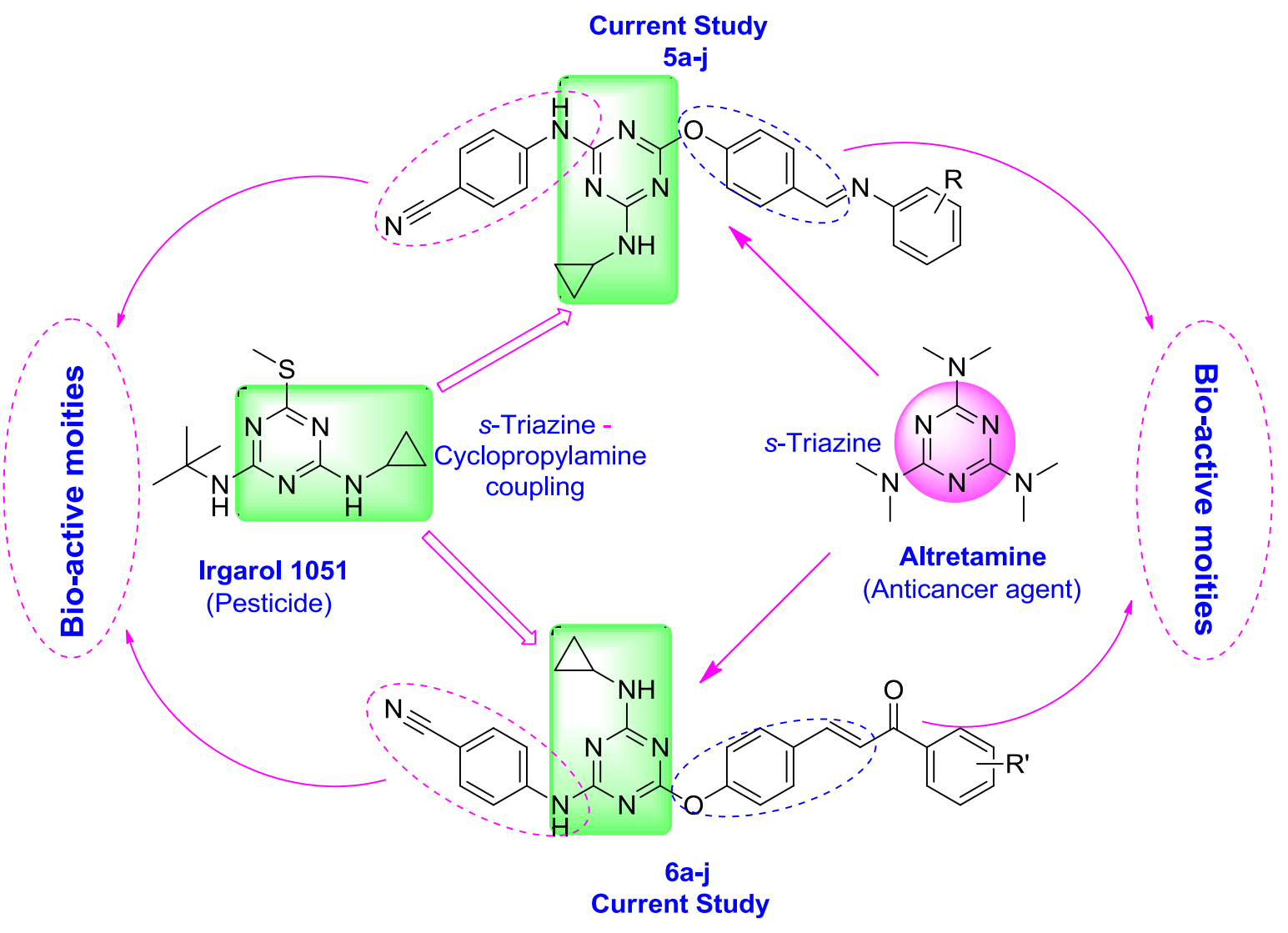

Figure 2. Rationalization of Titled Compounds.

$s$-Triazine derivatives represents an imperative group of drugs possessing miscellaneous medical applications by means of their widespread medicinal attribute such as antibacterial [4], antifungal [5], anti-TB [6], anticancer [7,8], antimalarial [9], anti-HIV [10] etc. Some triazine based drugs e.g. Altretamine, Triethylenemelamine and Atrazine (Fig. 1) have also been used for therapeutic purpose in clinic. Many chemists have synthesized effective antimicrobial agents containing $s$-triazine as a core moiety [11,12]. Schiff base possessing molecule having good inhibitory profile also been synthesized by many workers $[13,14]$. Triazine derivatives endowed with chalcone residue were also found to exhibit 
superior medicinal applications [15]. In this study, we have designed and developed a variety of $s$-triazine derivatives endowed with schiff base or chalcone residue [16-20].

\section{EXPERIMENTAL SECTION}

\section{1. Material and methods}

All the chemicals and solvents used for the synthesis work acquired from commercial sources were of analytical grade, and were used without further purification. Melting points were determined by using open capillary tubes and are uncorrected. TLC was checked on EMerck pre-coated 60 F254 plates and the spots were rendered visible by exposing to UV light or iodine. IR spectrums were recorded on SHIMADZU HYPER IR. NMR spectra were recorded by $400 \mathrm{MHz}$ BRUKER AVANCE instrument using TMS as internal standard (Chemical Shift in $\delta, \mathrm{ppm}$ ) and DMSO- $d 6$ as a solvent. Spectra were taken with a resonant frequency of $400 \mathrm{MHz}$ for ${ }^{1} \mathrm{H}$ and $100 \mathrm{MHz}$ for ${ }^{13} \mathrm{C}$ NMR. The splitting patterns are designated as follows; s, singlet; $\mathrm{d}$, doublet; dd, doublet of doublets; and $\mathrm{m}$, multiplet. Elemental analysis was done on "Haraeus Rapid Analyser". The mass spectra were recorded on JOEL SX-102 (EI) model with $60 \mathrm{eV}$ ionizing energy.

\section{Synthesis of 4-((4,6-dichloro-1,3,5-triazin-2-yl)amino)benzonitrile (2)}

To a solution of cyanuric chloride $(10 \mathrm{~g}, 0.054 \mathrm{~mol})$ in dry THF $(150 \mathrm{ml})$ at $0-5{ }^{\circ} \mathrm{C}$ were slowly added $\mathrm{K}_{2} \mathrm{CO}_{3}(14.9 \mathrm{~g}, 0.108 \mathrm{~mole})$ and 4-aminobenzonitrile $(6.41 \mathrm{~g}, 0.054$ mole). The solution was stirred for $4 \mathrm{hrs} 0-5{ }^{\circ} \mathrm{C}$. After completion of reaction based on TLC monitoring Toluene:Acetone (7:3), The resulted reaction mixture was then treated with crushed ice, followed by neutralization by dilute $\mathrm{HCl}$ and then filtered, dried and recrystallized from acetone to afford (2).

Yield- $79 \%$; mp 247-250 ${ }^{\circ} \mathrm{C}$; IR (vmax cm $\left.{ }^{-1}\right): 3277(\mathrm{~N}-\mathrm{H}$, secondary), $2228(\mathrm{C} \equiv \mathrm{N})$, $833\left(\mathrm{C}_{3} \mathrm{~N}_{3}, s\right.$-triazine), $802(\mathrm{C}-\mathrm{Cl}) ; 1 \mathrm{H}$ NMR (400 MHz, DMSO-d6, $\left.\delta \mathrm{ppm}\right): 8.61(\mathrm{~s}, 1 \mathrm{H},-$ $\mathrm{NH}$ 4-amino benzonitrile), 7.86-7.78 (m, 4H, Ar-H aromatic proton); 13C NMR (100 MHz, DMSO-d6, $\delta$ ppm): 171.72, 166.61, 165.35, 140.76, 132.17, 125.38, 122.61, 120.68, 119.32, 100.82; ESI-MS (m/z): $265.9(\mathrm{M}+)$.

\section{Synthesis of 4-((4-chloro-6-(cyclopropylamino)-1,3,5-triazin-2-yl)amino)benzonitrile (3)}

To a stirred solution of $2(5 \mathrm{~g}, 0.0188 \mathrm{~mol})$ in dry DMF $(20 \mathrm{~mL})$, slowly added a solution of cyclopropyl amine $(1.07 \mathrm{~g}, 0.0188 \mathrm{~mol})$ in DMF $(30 \mathrm{~mL})$ and $\mathrm{K}_{2} \mathrm{CO}_{3}(2.6 \mathrm{~g}$, $0.0188 \mathrm{~mol}$ ) at room temperature. The reaction mixture was stirred at room temperature for 9 hrs. After the completion of reaction, the reaction mass was dumped into crushed ice, solid was filtered, washed with water, dried to give crude product. The crude product was purified by column chromatography to get the pure product (3).

Yield- $70 \%$; mp $187-190{ }^{\circ} \mathrm{C}$; IR $\left(v \operatorname{max~cm} \mathrm{cm}^{-1}\right)$ : $3335(\mathrm{~N}-\mathrm{H}$, secondary), $2174(\mathrm{C} \equiv \mathrm{N})$, $821\left(\mathrm{C}_{3} \mathrm{~N}_{3}\right.$, s-triazine), $785(\mathrm{C}-\mathrm{Cl}) ;{ }^{1} \mathrm{H}$ NMR (400 MHz, DMSO-d6, $\left.\delta \mathrm{ppm}\right): 7.51$ (d, J = 7.5 $\mathrm{Hz}, 2 \mathrm{H}), 7.04(\mathrm{~d}, \mathrm{~J}=7.1 \mathrm{~Hz}, 2 \mathrm{H}), 6.85(\mathrm{~s}, 2 \mathrm{H}), 6.35(\mathrm{~s}, 1 \mathrm{H}), 4.80(\mathrm{~s}, 1 \mathrm{H}), 2.36(\mathrm{~s}, 3 \mathrm{H}), 2.23$ (s, 6H); ${ }^{13} \mathrm{C}$ NMR (100 MHz, DMSO-d6, $\delta$ ppm): 164.27, 162.56, 161.46, 145.27, 142.48, $135.55,133.49,131.13,129.57,128.84,127.74,114.24,22.25,19.16$; ESIMS (m/z): 365.8 $(\mathrm{M}+)$. 


\section{Synthesis of 4-((4-(cyclopropylamino)-6-(4-formylphenoxy)-1,3,5-triazin-2-yl)amino) benzonitrile (4)}

To a stirred solution of 3 (5 g, $0.0174 \mathrm{~mol})$ in dry DMF $(20 \mathrm{~mL})$, slowly added a solution of 4-hydroxy benzaldehyde $(2.13 \mathrm{~g}, 0.0174 \mathrm{~mol})$ in $\mathrm{DMF}(30 \mathrm{~mL})$ and $\mathrm{K}_{2} \mathrm{CO}_{3}(2.4 \mathrm{~g}$, $0.0174 \mathrm{~mol})$. After all the reagents have been added, the temperature of reaction raised upto $150{ }^{\circ} \mathrm{C}$. The reaction mass was refluxed for $11 \mathrm{hrs}$. After the completion of reaction, the reaction mass was cooled to room temperature, dumped into crushed ice, solid was filtered, washed with water and then dried to give crude product. It was then purified by crystallization from ethanol to give pure desired product (4).

Yield- $70 \%$; mp 153-157 ${ }^{\circ} \mathrm{C}$; IR (vmax cm $\left.{ }^{-1}\right): 3384(\mathrm{~N}-\mathrm{H}$, secondary), $2185(\mathrm{C} \equiv \mathrm{N})$, 1735 (Ar-CHO), $832\left(\mathrm{C}_{3} \mathrm{~N}_{3}\right.$, s-triazine); ${ }^{1} \mathrm{H}$ NMR (400 MHz, DMSO) $\delta 9.95(\mathrm{~s}, 1 \mathrm{H}), 7.66(\mathrm{~d}$, $J=7.43 \mathrm{~Hz}, 2 \mathrm{H}), 7.63(\mathrm{~d}, J=7.39 \mathrm{~Hz}, 2 \mathrm{H}), 7.30(\mathrm{~d}, J=7.7 \mathrm{~Hz}, 2 \mathrm{H}), 7.13(\mathrm{~d}, J=7.42 \mathrm{~Hz}$, 2H), $6.25(\mathrm{~s}, 1 \mathrm{H}), 3.54(\mathrm{~s}, 1 \mathrm{H}), 2.46(\mathrm{~m}, J=8.7 \mathrm{~Hz}, 1 \mathrm{H}), 1.71-1.52(\mathrm{~m}, 4 \mathrm{H}) ;{ }^{13} \mathrm{C}$ NMR $(100$ MHz, DMSO) $\delta 192.00,170.92,162.66,161.23,151.56,143.66,134.89,133.16,130.37$, 121.66, 119.41, 119.12, 100.31, 23.03, 8.93; ESIMS (m/z): $373.1(\mathrm{M}+)$.

\section{General procedure for the synthesis of compounds $5 \mathrm{a}-\mathrm{j}$}

A mixture of 4 (5 g, $0.0134 \mathrm{~mol})$, appropriate amine $(0.0134 \mathrm{~mol})$ and glacial acetic acid $(0.5 \mathrm{~mL})$ in ethanol $(50 \mathrm{~mL})$ was refluxed for $12 \mathrm{hrs}$. After the completion of reaction, the reaction mass was cooled to room temperature, dumped into crushed ice, solid was filtered, washed with water and then dried to give the desired crude product. The crude was then purified by column chromatography to get pure title compound.

\section{4-((4-(4-(((3-Chlorophenyl)imino)methyl)phenoxy)-6-(cyclopropylamino)-1,3,5-triazin-} 2-yl)amino)benzonitrile (5a)

IR (vmax cm $\left.{ }^{-1}\right): 3364\left(\mathrm{~N}-\mathrm{H}\right.$, secondary), $2174(\mathrm{C} \equiv \mathrm{N}), 825\left(\mathrm{C}_{3} \mathrm{~N}_{3}\right.$, s-triazine $) ;{ }^{1} \mathrm{H}$ NMR (400 MHz, DMSO) $\delta 8.74(\mathrm{~s}, 1 \mathrm{H}), 7.63-7.42(\mathrm{~m}, 5 \mathrm{H}), 7.32-7.10(\mathrm{~m}, 5 \mathrm{H}), 6.91-6.74(\mathrm{~m}, 2 \mathrm{H})$, $6.33(\mathrm{~s}, 1 \mathrm{H}), 3.43(\mathrm{~s}, 1 \mathrm{H}), 2.34-2.22(\mathrm{~m}, 1 \mathrm{H}), 1.34-0.24(\mathrm{~m}, 4 \mathrm{H}) ;{ }^{13} \mathrm{C}$ NMR $(100 \mathrm{MHz}$, DMSO) $\delta 171.45,163.56,160.23,150.44,145.47,144.23,135.55,134.74,131.39,130.75$, 126.30, 122.70, 121.80, 120.47, 118.56, 101.42, 24.27, 8.57; ESIMS (m/z): $482.5(\mathrm{M}+)$.

\section{4-((4-(4-(((4-Chlorophenyl)imino)methyl)phenoxy)-6-(cyclopropylamino)-1,3,5-triazin- 2-yl)amino)benzonitrile (5b)}

IR (vmax cm $\left.{ }^{-1}\right): 3360\left(\mathrm{~N}-\mathrm{H}\right.$, secondary), $2165(\mathrm{C} \equiv \mathrm{N}), 834\left(\mathrm{C}_{3} \mathrm{~N}_{3}\right.$, s-triazine $) ;{ }^{1} \mathrm{H}$ NMR (400 MHz, DMSO) $\delta 8.72(\mathrm{~s}, 1 \mathrm{H}), 7.50-7.36(\mathrm{~m}, 4 \mathrm{H}), 7.30-7.15(\mathrm{~m}, 4 \mathrm{H}), 7.11-7.02(\mathrm{~m}, 2 \mathrm{H})$, 6.96-6.71 (m, 2H), $6.20(\mathrm{~s}, 1 \mathrm{H}), 3.52(\mathrm{~s}, 1 \mathrm{H}), 2.45(\mathrm{~m}, 1 \mathrm{H}), 1.64-1.42(\mathrm{~m}, 4 \mathrm{H}) ;{ }^{13} \mathrm{C} \mathrm{NMR}$ $(100 \mathrm{MHz}, \mathrm{DMSO}) \delta 172.58,162.17,161.23,160.49,150.48,149.33,142.27,135.37$, $134.59,131.24,129.54,128.14,120.17,119.52,119.04,118.57,101.42,22.13,8.85$; ESIMS $(\mathrm{m} / \mathrm{z}): 482.4(\mathrm{M}+)$.

4-((4-(Cyclopropylamino)-6-(4-(((4-fluorophenyl)imino)methyl)phenoxy)-1,3,5-triazin-2yl)amino)benzonitrile (5c)

IR $\left(v \operatorname{vmax~cm}{ }^{-1}\right): 3372\left(\mathrm{~N}-\mathrm{H}\right.$, secondary), $2174(\mathrm{C} \equiv \mathrm{N}), 842\left(\mathrm{C}_{3} \mathrm{~N}_{3}\right.$, s-triazine $) ;{ }^{1} \mathrm{H}$ NMR (400 MHz, DMSO) $\delta 8.85(\mathrm{~s}, 1 \mathrm{H}), 7.62(\mathrm{~d}, J=7.44 \mathrm{~Hz}, 2 \mathrm{H}), 7.51(\mathrm{~d}, J=7.36 \mathrm{~Hz}, 2 \mathrm{H}), 7.51-$ $7.34(\mathrm{~m}, 2 \mathrm{H}), 7.30(\mathrm{~d}, J=7.28 \mathrm{~Hz}, 2 \mathrm{H}), 7.12(\mathrm{t}, J=7.7 \mathrm{~Hz}, 2 \mathrm{H}), 6.85(\mathrm{~d}, J=7.7 \mathrm{~Hz}, 2 \mathrm{H})$, 
$6.30(\mathrm{~s}, 1 \mathrm{H}), 3.62(\mathrm{~s}, 1 \mathrm{H}), 2.43-2.13(\mathrm{~m}, 1 \mathrm{H}), 1.62-1.20(\mathrm{~m}, 4 \mathrm{H}) ;{ }^{13} \mathrm{C} \mathrm{NMR}(100 \mathrm{MHz}$ DMSO) $\delta 172.43,162.53,161.22,160.37,159.33,158.04,152.34,151.13,144.37,135.27$, 134.43, 131.14, 123.39, 120.33, 119.65, 118.24, 117.46, 116.31, 102.47, 22.03, 8.56; ESIMS $(\mathrm{m} / \mathrm{z}): 466.3(\mathrm{M}+)$.

4-((4-(Cyclopropylamino)-6-(4-(((3-nitrophenyl)imino)methyl)phenoxy)-1,3,5-triazin-2yl)amino)benzonitrile (5d)

IR (vmax cm $\left.{ }^{-1}\right): 3292(\mathrm{~N}-\mathrm{H}$, secondary), $2218(\mathrm{C} \equiv \mathrm{N}), 1521(\mathrm{~N}-\mathrm{O}$ stretch), 1355 (N-O stretch), $839\left(\mathrm{C}_{3} \mathrm{~N}_{3}\right.$, s-triazine); ${ }^{1} \mathrm{H}$ NMR (400 MHz, DMSO) $\delta 8.88(\mathrm{~s}, 1 \mathrm{H}), 8.59-8.57(\mathrm{~m}$, $3 \mathrm{H}), 8.41-7.83(\mathrm{~m}, 5 \mathrm{H}), 7.58-7.37(\mathrm{~m}, 4 \mathrm{H}), 6.07(\mathrm{~s}, 1 \mathrm{H}), 3.81(\mathrm{~s}, 1 \mathrm{H}), 3.11-2.80(\mathrm{~m}, 1 \mathrm{H})$, $1.20-0.75(\mathrm{~m}, 4 \mathrm{H}) ;{ }^{13} \mathrm{C}$ NMR $(100 \mathrm{MHz}, \mathrm{DMSO}) \delta 170.15,162.60,161.12,153.67,152.08$, $148.43,144.07,135.12$, 132.51, 130.30, 127.91, 126.13, 125.46, 124.17, 123.61, 122.02, 121.54, 120.65, 119.23, 101.26, 24.64, 8.57; ESIMS (m/z): $493.4(\mathrm{M}+)$.

\section{4-((4-(Cyclopropylamino)-6-(4-(((4-nitrophenyl)imino)methyl)phenoxy)-1,3,5-triazin-2- yl)amino)benzonitrile (5e)}

IR (vmax cm $\left.{ }^{-1}\right): 3385(\mathrm{~N}-\mathrm{H}$, secondary), $2166(\mathrm{C} \equiv \mathrm{N}), 1545(\mathrm{~N}-\mathrm{O}$ stretch), $1367(\mathrm{~N}-\mathrm{O}$ stretch), $845\left(\mathrm{C}_{3} \mathrm{~N}_{3}\right.$, s-triazine); ${ }^{1} \mathrm{H}$ NMR (400 MHz, DMSO) $\delta 8.75(\mathrm{~s}, 1 \mathrm{H}), 8.20(\mathrm{~d}, J=7.7$ $\mathrm{Hz}, 2 \mathrm{H}), 7.79$ (d, $J=7.42 \mathrm{~Hz}, 2 \mathrm{H}), 7.61$ (dd, $J=17.5,7.5 \mathrm{~Hz}, 4 \mathrm{H}), 7.25$ (d, $J=7.1 \mathrm{~Hz}, 2 \mathrm{H})$, $6.90(\mathrm{~d}, J=7.54 \mathrm{~Hz}, 2 \mathrm{H}), 6.31(\mathrm{~s}, 1 \mathrm{H}), 3.62(\mathrm{~s}, 1 \mathrm{H}), 2.45-2.24(\mathrm{~m}, 1 \mathrm{H}), 1.21-0.74(\mathrm{~m}, 4 \mathrm{H})$; ${ }^{13} \mathrm{C}$ NMR (100 MHz, DMSO) $\delta 170.90,163.12,162.60,161.15,158.12,151.43,144.25$, $143.62,134.50,133.64,130.32,125.25,121.49,120.74,119.05,100.26,23.21,8.80$; ESIMS $(\mathrm{m} / \mathrm{z}): 493.5(\mathrm{M}+)$.

\section{4-((4-(Cyclopropylamino)-6-(4-(((3-hydroxyphenyl)imino)methyl)phenoxy)-1,3,5-} triazin-2-yl)amino)benzonitrile (5f)

IR (vmax cm $\left.{ }^{-1}\right): 3427(\mathrm{O}-\mathrm{H}$ broad band), $3314(\mathrm{~N}-\mathrm{H}$, secondary), $2176(\mathrm{C} \equiv \mathrm{N}), 1526$ (N-O stretch), 1374 (N-O stretch), $856\left(\mathrm{C}_{3} \mathrm{~N}_{3}\right.$, s-triazine); ${ }^{1} \mathrm{H}$ NMR (400 MHz, DMSO) $\delta 8.84$ $(\mathrm{s}, 1 \mathrm{H}), 8.25(\mathrm{~s}, 1 \mathrm{H}), 7.46(\mathrm{dd}, J=27.1,7.5 \mathrm{~Hz}, 4 \mathrm{H}), 7.27(\mathrm{~d}, J=7.37 \mathrm{~Hz}, 2 \mathrm{H}), 7.12(\mathrm{t}, J=$ $7.33 \mathrm{~Hz}, 1 \mathrm{H}), 6.86-6.77(\mathrm{~m}, 4 \mathrm{H}), 6.75-6.45(\mathrm{~m}, 1 \mathrm{H}), 6.28(\mathrm{~s}, 1 \mathrm{H}), 3.43(\mathrm{~s}, 1 \mathrm{H}), 2.46-2.28(\mathrm{~m}$, 1H), 2.18-0.87 (m, 4H); ${ }^{13} \mathrm{C}$ NMR (100 MHz, DMSO) $\delta 170.80,162.54,161.12,158.61$, $151.43,151.10,143.74,134.62$, 133.75, 130.94, 130.31, 121.64, 120.82, 119.10, 114.70, 113.22, 108.61, 100.24, 23.14, 8.84; ESIMS (m/z): $464.1(\mathrm{M}+)$.

\section{4-((4-(Cyclopropylamino)-6-(4-(((4-hydroxyphenyl)imino)methyl)phenoxy)-1,3,5- triazin-2-yl)amino)benzonitrile $(5 \mathrm{~g})$}

IR (vmax cm $\left.{ }^{-1}\right): 3456(\mathrm{O}-\mathrm{H}$ broad band), $3327(\mathrm{~N}-\mathrm{H}$, secondary), $2153(\mathrm{C} \equiv \mathrm{N}), 1546$ (N-O stretch), 1380 (N-O stretch), $844\left(\mathrm{C}_{3} \mathrm{~N}_{3}\right.$, s-triazine); ${ }^{1} \mathrm{H}$ NMR (400 MHz, DMSO) $\delta 8.73$ $(\mathrm{s}, 1 \mathrm{H}), 8.42(\mathrm{~s}, 1 \mathrm{H}), 7.46(\mathrm{~d}, J=7.52 \mathrm{~Hz}, 2 \mathrm{H}), 7.38(\mathrm{~d}, J=7.41 \mathrm{~Hz}, 2 \mathrm{H}), 7.13(\mathrm{dd}, J=12.3$, $7.6 \mathrm{~Hz}, 4 \mathrm{H}), 6.90(\mathrm{~d}, J=7.33 \mathrm{~Hz}, 2 \mathrm{H}), 6.73(\mathrm{~d}, J=7.45 \mathrm{~Hz}, 2 \mathrm{H}), 6.25(\mathrm{~s}, 1 \mathrm{H}), 3.53(\mathrm{~s}, 1 \mathrm{H})$, 2.42-2.35 (m, 1H), 2.10-0.76 (m, 4H); ${ }^{13} \mathrm{C}$ NMR (100 MHz, DMSO) $\delta ~ 170.98, ~ 163.64$, $162.43,161.34,158.27,151.18,144.82,143.74,134.67,133.43,130.29,122.56,121.43$, 120.75, 119.09, 116.76, 100.29, 23.15, 8.82; ESIMS (m/z): $464.2(\mathrm{M}+)$. 


\section{4-((4-(4-((Cyclohexylimino)methyl)phenoxy)-6-(cyclopropylamino)-1,3,5-triazin-2- yl)amino)benzonitrile (5h)}

IR (vmax cm $\left.{ }^{-1}\right): 3346(\mathrm{~N}-\mathrm{H}$, secondary), 2954 (alkyl C-H stretch), $2157(\mathrm{C} \equiv \mathrm{N}), 847$ $\left(\mathrm{C}_{3} \mathrm{~N}_{3}\right.$, s-triazine); ${ }^{1} \mathrm{H}$ NMR (400 MHz, DMSO) $\delta 8.24(\mathrm{~s}, 1 \mathrm{H}), 7.43(\mathrm{~d}, J=7.27 \mathrm{~Hz}, 2 \mathrm{H})$, $7.32(\mathrm{~d}, J=7.54 \mathrm{~Hz}, 2 \mathrm{H}), 7.15(\mathrm{~d}, J=7.4 \mathrm{~Hz}, 2 \mathrm{H}), 6.90(\mathrm{~d}, J=7.6 \mathrm{~Hz}, 2 \mathrm{H}), 6.22(\mathrm{~s}, 1 \mathrm{H})$, $3.63(\mathrm{~s}, 1 \mathrm{H}), 2.83-2.71(\mathrm{~m}, 1 \mathrm{H}), 2.40-2.21(\mathrm{~m}, 1 \mathrm{H}), 1.86(\mathrm{dt}, J=7.6,5.8 \mathrm{~Hz}, 2 \mathrm{H}), 1.81-1.40$ $(\mathrm{m}, 7 \mathrm{H}), 1.30-1.17(\mathrm{~m}, 1 \mathrm{H}), 1.05-0.22(\mathrm{~m}, 4 \mathrm{H}) ;{ }^{13} \mathrm{C}$ NMR $(100 \mathrm{MHz}, \mathrm{DMSO}) \delta 170.80$, $162.75,162.23,161.12,150.30,143.54,134.40,133.13,130.30,121.52,120.90,119.25$, $100.23,66.42,33.73,25.96,25.33,23.24,8.65$; ESIMS (m/z): $454.3(\mathrm{M}+)$.

\section{4-((4-(Cyclopropylamino)-6-(4-((o-tolylimino)methyl)phenoxy)-1,3,5-triazin-2- yl)amino)benzonitrile (5i)}

IR (vmax cm $\left.{ }^{-1}\right): 3348\left(\mathrm{~N}-\mathrm{H}\right.$, secondary), $2163(\mathrm{C} \equiv \mathrm{N}), 849\left(\mathrm{C}_{3} \mathrm{~N}_{3}\right.$, s-triazine $) ;{ }^{1} \mathrm{H}$ NMR (400 MHz, DMSO) $\delta 8.77(\mathrm{~s}, 1 \mathrm{H}), 7.64(\mathrm{~d}, J=7.5 \mathrm{~Hz}, 2 \mathrm{H}), 7.49(\mathrm{~d}, J=7.53 \mathrm{~Hz}, 2 \mathrm{H}), 7.41-$ $6.54(\mathrm{~m}, 8 \mathrm{H}), 6.26(\mathrm{~s}, 1 \mathrm{H}), 3.63(\mathrm{~s}, 1 \mathrm{H}), 2.42-2.25(\mathrm{~m}, 4 \mathrm{H}), 1.63-1.13(\mathrm{~m}, 4 \mathrm{H}) ;{ }^{13} \mathrm{C}$ NMR $(100 \mathrm{MHz}, \mathrm{DMSO}) \delta 170.96,162.63,161.34,155.19,151.27,150.43,143.50,134.61$, $133.73,130.29,129.65,129.22,127.70,126.52,121.54,120.72,120.17,119.44,100.47$, 23.23, 18.46, 8.66; ESIMS (m/z): $462.6(\mathrm{M}+)$.

\section{(4-((4-(Cyclopropylamino)-6-(4-((p-tolylimino)methyl)phenoxy)-1,3,5-triazin-2-} yl)amino)benzonitrile (5j)

IR (vmax cm $\left.{ }^{-1}\right): 3357\left(\mathrm{~N}-\mathrm{H}\right.$, secondary), $2138(\mathrm{C} \equiv \mathrm{N}), 851\left(\mathrm{C}_{3} \mathrm{~N}_{3}\right.$, s-triazine $) ;{ }^{1} \mathrm{H}$ NMR (400 MHz, DMSO) $\delta 8.71(\mathrm{~s}, 1 \mathrm{H}), 7.49(\mathrm{dd}, J=14.1,7.7 \mathrm{~Hz}, 4 \mathrm{H}), 7.32(\mathrm{~d}, J=7.43 \mathrm{~Hz}, 2 \mathrm{H})$, $7.20(\mathrm{dd}, J=13.1,7.38 \mathrm{~Hz}, 4 \mathrm{H}), 6.95(\mathrm{~d}, J=7.58 \mathrm{~Hz}, 2 \mathrm{H}), 6.53(\mathrm{~s}, 1 \mathrm{H}), 3.60(\mathrm{~s}, 1 \mathrm{H}), 2.47-$ $2.31(\mathrm{~m}, 4 \mathrm{H}), 1.82-0.69(\mathrm{~m}, 4 \mathrm{H}) ;{ }^{13} \mathrm{C}$ NMR $(100 \mathrm{MHz}, \mathrm{DMSO}) \delta 170.77,163.13,162.57$, $161.35,151.18,150.43,143.12,134.43,133.47,130.58,129.24,121.45,120.75,119.25$, 100.37, 23.42, 21.13, 8.52; ESIMS (m/z): $462.3(\mathrm{M}+)$.

\section{General procedure for the synthesis of compounds $6 \mathrm{a}-\mathrm{j}$}

A mixture of 4 ( $5 \mathrm{~g}, 0.0134 \mathrm{~mol})$, appropriate acetophenone $(0.0134 \mathrm{~mol})$ and sodium hydroxide $(0.51 \mathrm{~g}, 0.0147 \mathrm{~mol})$ in methanol $(50 \mathrm{~mL})$ was refluxed for $15 \mathrm{hrs}$. After the completion of reaction, the reaction mass was cooled to room temperature, dumped into crushed ice, solid was filtered, washed with water and then dried to give the desired crude product. The crude was then purified by column chromatography to get pure title compound.

\section{4-((4-(Cyclopropylamino)-6-(4-(3-oxo-3-phenylprop-1-en-1-yl)phenoxy)-1,3,5-triazin-2-} yl)amino)benzonitrile (6a)

IR (vmax cm $\left.{ }^{-1}\right): 3325(\mathrm{~N}-\mathrm{H}$, secondary), $2220(\mathrm{C} \equiv \mathrm{N}), 1635$ (alkene $\mathrm{C}=\mathrm{C}$ stretch), 839 $\left(\mathrm{C}_{3} \mathrm{~N}_{3}\right.$, s-triazine); ${ }^{1} \mathrm{H}$ NMR (400 MHz, DMSO) $\delta$ 8.17-8.13 (m, 2H), 7.98-7.73 (m, 6H), 7.67-7.38 (m, 3H), 7.32-7.14 (m, 4H), $6.20(\mathrm{~s}, 1 \mathrm{H}), 3.42(\mathrm{~s}, 1 \mathrm{H}), 2.20-2.01(\mathrm{~m}, 1 \mathrm{H}), 0.73-$ $0.65(\mathrm{~m}, 2 \mathrm{H}), 0.56-0.43(\mathrm{~m}, 2 \mathrm{H}) ;{ }^{13} \mathrm{C}$ NMR (100 MHz, DMSO) $\delta 191.18,169.33,163.85$, 161.32 , 151.65, 143.36, 139.58, 131.84, 129.05, 128.83, 126.32, 125.50, 124.11, 123.36, $122.15,121.36,120.22,119.53,100.18,22.16,8.83$; ESIMS (m/z): $475.8(\mathrm{M}+)$. 
4-((4-(4-(3-(2-Chlorophenyl)-3-oxoprop-1-en-1-yl)phenoxy)-6-(cyclopropylamino)-1,3,5triazin-2-yl)amino)benzonitrile (6b)

IR (vmax cm $\left.{ }^{-1}\right): 3337(\mathrm{~N}-\mathrm{H}$, secondary), $2148(\mathrm{C} \equiv \mathrm{N}), 1640$ (alkene $\mathrm{C}=\mathrm{C}$ stretch), 1035 (ether C-O stretch), $843\left(\mathrm{C}_{3} \mathrm{~N}_{3}\right.$, s-triazine); ${ }^{1} \mathrm{H}$ NMR (400 MHz, DMSO) $\delta$ 7.81-7.63 (m, $\left.2 \mathrm{H}\right)$, 7.54-7.45 (m, 5H), 7.40-7.26 (m, 1H), $7.16(\mathrm{dd}, J=7.3,6.8 \mathrm{~Hz}, 4 \mathrm{H}), 6.84(\mathrm{~d}, J=7.2 \mathrm{~Hz}$, $2 \mathrm{H}), 6.21(\mathrm{~s}, 1 \mathrm{H}), 3.65(\mathrm{~s}, 1 \mathrm{H}), 2.46-2.32(\mathrm{~m}, 1 \mathrm{H}), 1.98-1.25(\mathrm{~m}, 4 \mathrm{H}) ;{ }^{13} \mathrm{C}$ NMR $(100 \mathrm{MHz}$, DMSO) $\delta 189.35,170.62,162.57,161.12,151.55,143.46,143.73,138.16,135.54,133.42$, $131.47,131.58,130.28,130.67,129.86,126.23,122.68,121.78,120.38,119.66,100.87$, 23.46, 8.28; ESIMS (m/z): $509.6(\mathrm{M}+)$.

4-((4-(4-(3-(4-Chlorophenyl)-3-oxoprop-1-en-1-yl)phenoxy)-6-(cyclopropylamino)-1,3,5triazin-2-yl)amino)benzonitrile (6c)

IR (vmax cm $\left.{ }^{-1}\right): 3327(\mathrm{~N}-\mathrm{H}$, secondary), $2141(\mathrm{C} \equiv \mathrm{N}), 1643$ (alkene $\mathrm{C}=\mathrm{C}$ stretch), 1143 (ether C-O stretch), $856\left(\mathrm{C}_{3} \mathrm{~N}_{3}\right.$, s-triazine); ${ }^{1} \mathrm{H}$ NMR (400 MHz, DMSO) $\delta 7.70$ (dd, $J=11.3$, $7.2 \mathrm{~Hz}, 3 \mathrm{H}), 7.60-7.42(\mathrm{~m}, 5 \mathrm{H}), 7.30(\mathrm{dd}, J=7.4,6.9 \mathrm{~Hz}, 4 \mathrm{H}), 6.75(\mathrm{~d}, J=7.37 \mathrm{~Hz}, 2 \mathrm{H}), 6.28$ $(\mathrm{s}, 1 \mathrm{H}), 3.65(\mathrm{~s}, 1 \mathrm{H}), 2.47-2.29(\mathrm{~m}, 1 \mathrm{H}), 2.15-1.43(\mathrm{~m}, 4 \mathrm{H}) ;{ }^{13} \mathrm{C}$ NMR $(100 \mathrm{MHz}, \mathrm{DMSO}) \delta$ $190.58,170.67,162.48,161.38,151.57,144.49,143.15,138.78,137.49,131.15,131.26$, 130.48, 129.67, 129.34, 122.67, 121.34, 120.21, 119.25, 100.47, 23.69, 8.58; ESIMS (m/z): $509.5(\mathrm{M}+)$.

\section{4-((4-(Cyclopropylamino)-6-(4-(3-(2,5-dichlorophenyl)-3-oxoprop-1-en-1-yl)phenoxy)-} 1,3,5-triazin-2-yl)amino)benzonitrile (6d)

IR (vmax cm $\left.{ }^{-1}\right): 3364(\mathrm{~N}-\mathrm{H}$, secondary), $2154(\mathrm{C} \equiv \mathrm{N}), 1637$ (alkene $\mathrm{C}=\mathrm{C}$ stretch), 1143 (ether C-O stretch), $839\left(\mathrm{C}_{3} \mathrm{~N}_{3}\right.$, s-triazine); ${ }^{1} \mathrm{H}$ NMR (400 MHz, DMSO) $\delta 7.74(\mathrm{~d}, J=1.2$ $\mathrm{Hz}, 1 \mathrm{H}), 7.59(\mathrm{~d}, J=14.9 \mathrm{~Hz}, 1 \mathrm{H}), 7.52-7.35(\mathrm{~m}, 5 \mathrm{H}), 7.28-7.14(\mathrm{~m}, 4 \mathrm{H}), 6.75(\mathrm{~d}, J=7.6 \mathrm{~Hz}$, $2 \mathrm{H}), 6.36(\mathrm{~s}, 1 \mathrm{H}), 3.64(\mathrm{~s}, 1 \mathrm{H}), 2.48-1.76(\mathrm{~m}, 1 \mathrm{H}), 1.54-0.82(\mathrm{~m}, 4 \mathrm{H}) ;{ }^{13} \mathrm{C}$ NMR $(100 \mathrm{MHz}$, DMSO) $\delta 189.55,170.82,162.37,161.45,151.73,131.54,143.42,143.75,142.00,140.27$, $132.23,131.45,130.15,122.43,121.37,120.85,119.43,100.18,23.73,8.49 ;$ ESIMS (m/z): $544.8(\mathrm{M}+)$.

4-((4-(Cyclopropylamino)-6-(4-(3-oxo-3-(o-tolyl)prop-1-en-1-yl)phenoxy)-1,3,5-triazin-2yl)amino)benzonitrile (6e)

IR (vmax cm $\left.{ }^{-1}\right): 3359(\mathrm{~N}-\mathrm{H}$, secondary), $2137(\mathrm{C} \equiv \mathrm{N}), 1655$ (alkene $\mathrm{C}=\mathrm{C}$ stretch), 1059 (ether C-O stretch), $852\left(\mathrm{C}_{3} \mathrm{~N}_{3}\right.$, s-triazine); ${ }^{1} \mathrm{H}$ NMR (400 MHz, DMSO) $\delta$ 7.85-7.68 (m, 2H), 7.60-7.48 (m, 4H), 7.40-7.27 (m, 2H), 7.20-7.08 (m, 4H), $6.92(\mathrm{~d}, J=7.1 \mathrm{~Hz}, 2 \mathrm{H}), 6.35(\mathrm{~s}$, $1 \mathrm{H}), 3.74(\mathrm{~s}, 1 \mathrm{H}), 2.37-2.21(\mathrm{~m}, 4 \mathrm{H}), 1.24-1.07(\mathrm{~m}, 2 \mathrm{H}), 0.94-0.49(\mathrm{~m}, 2 \mathrm{H}) ;{ }^{13} \mathrm{C}$ NMR $(100$ MHz, DMSO) $\delta 193.17,170.52,162.73,161.64,151.82,143.37,143.49,139.57,137.36$, $132.15,131.46,131.37,131.28,130.53,129.42,126.37,122.41,121.55,120.76,119.35$, 100.12, 23.43, 20.75, 8.36; ESIMS (m/z): $489.3(\mathrm{M}+)$.

4-((4-(Cyclopropylamino)-6-(4-(3-(2-nitrophenyl)-3-oxoprop-1-en-1-yl)phenoxy)-1,3,5triazin-2-yl)amino)benzonitrile (6f)

IR (vmax cm $\left.{ }^{-1}\right): 3349(\mathrm{~N}-\mathrm{H}$, secondary), $2134(\mathrm{C} \equiv \mathrm{N}), 1675$ (alkene $\mathrm{C}=\mathrm{C}$ stretch), 1150 (ether C-O stretch), $853\left(\mathrm{C}_{3} \mathrm{~N}_{3}\right.$, s-triazine); ${ }^{1} \mathrm{H}$ NMR (400 MHz, DMSO) $\delta$ 9.10-7.42 (m, 2H), $7.75(\mathrm{~m}, 2 \mathrm{H}), 7.46(\mathrm{~m}, 1 \mathrm{H}), 7.40(\mathrm{~m}, 3 \mathrm{H}), 7.26(\mathrm{~m}, 4 \mathrm{H}), 6.73(\mathrm{~d}, J=7.8 \mathrm{~Hz}, 2 \mathrm{H}), 6.34(\mathrm{~s}$, $1 \mathrm{H}), 3.57(\mathrm{~s}, 1 \mathrm{H}), 2.58-2.35(\mathrm{~m}, 1 \mathrm{H}), 2.11-1.37(\mathrm{~m}, 4 \mathrm{H}) ;{ }^{13} \mathrm{C}$ NMR (100 MHz, DMSO) $\delta$ 
$194.15,170.72,162.64,161.63,151.58,150.73,143.42,143.11,133.37,132.42,131.15$, $131.43,130.85,128.73,122.62,122.46,121.75,120.42,119.64,100.33,23.42,8.58$; ESIMS $(\mathrm{m} / \mathrm{z}): 520.4(\mathrm{M}+)$.

4-((4-(Cyclopropylamino)-6-(4-(3-(2-hydroxy-5-nitrophenyl)-3-oxoprop-1-en-1yl)phenoxy)-1,3,5-triazin-2-yl)amino)benzonitrile (6g)

IR (vmax cm $\left.{ }^{-1}\right): 3452(\mathrm{O}-\mathrm{H}$ broad band), $3364(\mathrm{~N}-\mathrm{H}$, secondary), $2176(\mathrm{C} \equiv \mathrm{N}), 1664$ (alkene $\mathrm{C}=\mathrm{C}$ stretch), 1556 (N-O stretch), 1360 (N-O stretch), 1184 (ether C-O stretch), 863 $\left(\mathrm{C}_{3} \mathrm{~N}_{3}\right.$, s-triazine); ${ }^{1} \mathrm{H}$ NMR $(400 \mathrm{MHz}, \mathrm{DMSO}) \delta 9.62(\mathrm{~s}, 1 \mathrm{H}), 8.63(\mathrm{~d}, J=1.4 \mathrm{~Hz}, 1 \mathrm{H}), 8.40$ $(\mathrm{dd}, J=7.5,1.4 \mathrm{~Hz}, 1 \mathrm{H}), 7.82(\mathrm{~d}, J=14.9 \mathrm{~Hz}, 1 \mathrm{H}), 7.62(\mathrm{t}, J=11.2 \mathrm{~Hz}, 3 \mathrm{H}), 7.34(\mathrm{dd}, J=$ 7.3, 5.4 Hz, 5H), $6.72(\mathrm{~d}, J=7.3 \mathrm{~Hz}, 2 \mathrm{H}), 6.37(\mathrm{~s}, 1 \mathrm{H}), 3.43(\mathrm{~s}, 1 \mathrm{H}), 2.44-2.38(\mathrm{~m}, 1 \mathrm{H}), 2.53-$ $1.24(\mathrm{~m}, 4 \mathrm{H}) ;{ }^{13} \mathrm{C}$ NMR (100 MHz, DMSO) $\delta$ 192.53, 170.27, 169.43, 162.51, 161.34, 151.52 , 143.82, 143.14, 138.30, 131.24, 131.87, 130.50, 126.42, 124.13, 122.22, 121.08, 120.63, 119.74, 119.23, 100.04, 23.37, 8.42; ESIMS (m/z): $536.7(\mathrm{M}+)$.

4-((4-(4-(3-(5-Chloro-2-hydroxy-3-nitrophenyl)-3-oxoprop-1-en-1-yl)phenoxy)-6(cyclopropylamino)-1,3,5-triazin-2-yl)amino)benzonitrile (6h)

IR (vmax cm $\left.{ }^{-1}\right): 3521(\mathrm{O}-\mathrm{H}$ broad band), $3387(\mathrm{~N}-\mathrm{H}$, secondary), $2157(\mathrm{C} \equiv \mathrm{N}), 1678$ (alkene $\mathrm{C}=\mathrm{C}$ stretch), 1563 (N-O stretch), 1358 (N-O stretch), 1192 (ether C-O stretch), 860 $\left(\mathrm{C}_{3} \mathrm{~N}_{3}\right.$, s-triazine); ${ }^{1} \mathrm{H}$ NMR (400 MHz, DMSO) $\delta 13.23(\mathrm{~s}, 1 \mathrm{H}), 8.34(\mathrm{~d}, J=1.4 \mathrm{~Hz}, 1 \mathrm{H})$, $8.13(\mathrm{~d}, J=1.6 \mathrm{~Hz}, 1 \mathrm{H}), 7.54(\mathrm{~d}, J=15.1 \mathrm{~Hz}, 1 \mathrm{H}), 7.46(\mathrm{dd}, J=12.5,11.4 \mathrm{~Hz}, 3 \mathrm{H}), 7.31$ (dd, $J=7.3,6.8 \mathrm{~Hz}, 4 \mathrm{H}), 6.52(\mathrm{~d}, J=7.7 \mathrm{~Hz}, 2 \mathrm{H}), 6.37(\mathrm{~s}, 1 \mathrm{H}), 3.40(\mathrm{~s}, 1 \mathrm{H}), 2.37-1.92(\mathrm{~m}, 1 \mathrm{H})$, 1.73-1.24 (m, 4H); ${ }^{13} \mathrm{C}$ NMR (100 MHz, DMSO) $\delta 192.67,170.48,162.29,161.49,155.67$, 151.32 , 143.45, 143.64, 136.32, 135.18, 131.43, 131.73, 130.46, 127.44, 125.75, 122.81, 121.94, 120.67, 119.78, 100.32, 23.44, 8.75; ESIMS (m/z): $570.6(\mathrm{M}+)$.

4-((4-(Cyclopropylamino)-6-(4-(3-(5-fluoro-2-hydroxy-3-nitrophenyl)-3-oxoprop-1-en-1yl)phenoxy)-1,3,5-triazin-2-yl)amino)benzonitrile (6i)

IR (vmax cm $\left.{ }^{-1}\right): 3467(\mathrm{O}-\mathrm{H}$ broad band), $3351(\mathrm{~N}-\mathrm{H}$, secondary), $2149(\mathrm{C} \equiv \mathrm{N}), 1674$ (alkene $\mathrm{C}=\mathrm{C}$ stretch), 1559 (N-O stretch), 1348 (N-O stretch), 1183 (ether C-O stretch), 854 $\left(\mathrm{C}_{3} \mathrm{~N}_{3}\right.$, s-triazine); ${ }^{1} \mathrm{H}$ NMR (400 MHz, DMSO) $\delta 13.11(\mathrm{~s}, 1 \mathrm{H}), 8.42(\mathrm{dd}, J=8.0,1.5 \mathrm{~Hz}$, 1H), 7.45 (dd, $J=7.9,1.4 \mathrm{~Hz}, 1 \mathrm{H}), 7.37$ (d, $J=15.1 \mathrm{~Hz}, 1 \mathrm{H}), 7.40$ (t, $J=10.9 \mathrm{~Hz}, 3 \mathrm{H}), 7.75$ $(\mathrm{t}, J=7.7 \mathrm{~Hz}, 4 \mathrm{H}), 6.67(\mathrm{~d}, J=7.7 \mathrm{~Hz}, 2 \mathrm{H}), 6.11(\mathrm{~s}, 1 \mathrm{H}), 3.70(\mathrm{~s}, 1 \mathrm{H}), 2.43-1.89(\mathrm{~m}, 1 \mathrm{H})$, $1.71-1.10(\mathrm{~m}, 4 \mathrm{H}) ;{ }^{13} \mathrm{C}$ NMR (100 MHz, DMSO) $\delta 192.40,170.35,162.41,161.43,160.12$, $157.36,154.10,151.22,143.43,143.57,136.42,131.58,131.10,130.54,126.63,124.67$, $124.28,122.75,121.62,120.18,119.61,117.64,117.52,100.31,23.48,8.61 ;$ ESIMS (m/z): $554.9(\mathrm{M}+)$.

4-((4-(Cyclopropylamino)-6-(4-(3-(2,4-dihydroxy-3-methyl-5-nitrophenyl)-3-oxoprop-1en-1-yl)phenoxy)-1,3,5-triazin-2-yl)amino)benzonitrile (6j)

IR (vmax cm $\left.{ }^{-1}\right): 3487(\mathrm{O}-\mathrm{H}$ broad band), $3373(\mathrm{~N}-\mathrm{H}$, secondary), $2168(\mathrm{C} \equiv \mathrm{N}), 1666$ (alkene $\mathrm{C}=\mathrm{C}$ stretch), 1554 (N-O stretch), 1351 (N-O stretch), 1183 (ether C-O stretch), 852 $\left(\mathrm{C}_{3} \mathrm{~N}_{3}\right.$, s-triazine); ${ }^{1} \mathrm{H}$ NMR $(400 \mathrm{MHz}, \mathrm{DMSO}) \delta 9.34(\mathrm{~s}, 1 \mathrm{H}), 9.24(\mathrm{~s}, 1 \mathrm{H}), 8.31(\mathrm{~s}, 1 \mathrm{H})$, $7.80(\mathrm{~d}, J=15.1 \mathrm{~Hz}, 1 \mathrm{H}), 7.46-7.27(\mathrm{~m}, 3 \mathrm{H}), 7.19-7.04(\mathrm{~m}, 4 \mathrm{H}), 6.65(\mathrm{~d}, J=7.35 \mathrm{~Hz}, 2 \mathrm{H})$, $6.37(\mathrm{~s}, 1 \mathrm{H}), 3.37(\mathrm{~s}, 1 \mathrm{H}), 2.43-1.84(\mathrm{~m}, 4 \mathrm{H}), 1.60-1.36(\mathrm{~m}, 4 \mathrm{H}) ;{ }^{13} \mathrm{C}$ NMR $(100 \mathrm{MHz}$, DMSO) $\delta 192.33,170.75,167.43,162.67,161.10,156.54,151.63,143.84,143.42,131.64$, 
$131.35,130.45,125.73,125.27,122.95,121.60,120.55,119.35,114.67,110.18,100.25$, 23.34, 8.93, 8.47; ESIMS (m/z): $566.4(\mathrm{M}+)$.

Table 1. In-vitro anti-bacterial activity of newly synthesized compounds $\mathbf{5 a - j}$ and $\mathbf{6 a - j}$.

\begin{tabular}{|c|c|c|c|c|c|c|c|}
\hline \multirow[b]{2}{*}{ Entry } & \multirow[b]{2}{*}{$\mathbf{R} / \mathbf{R}^{\prime}$} & \multirow[b]{2}{*}{$\log P$} & \multicolumn{5}{|c|}{ Zone of Inhibition in $\mathrm{mm}$ (MIC in $\mu \mathrm{g} / \mathrm{mL}$ ) } \\
\hline & & & S.a & B.s & E.c & $P . a$ & K.p \\
\hline $5 a$ & & 7.03 & $21(6.25)$ & $27(100)$ & $19(200)$ & $25(100)$ & $15(50)$ \\
\hline $5 \mathbf{b}$ & & 7.03 & $19(6.25)$ & $30(50)$ & $13(50)$ & $20(100)$ & $11(100)$ \\
\hline $5 c$ & & 6.63 & $11(50)$ & $25(200)$ & $24(6.25)$ & $16(25)$ & 09 (12.5) \\
\hline $5 d$ & & - & $15(100)$ & $26(50)$ & $17(100)$ & $17(50)$ & $14(50)$ \\
\hline $5 e$ & & - & $08(12.5)$ & $22(12.5)$ & $20(12.5)$ & $21(100)$ & $10(200)$ \\
\hline $\mathbf{5 f}$ & & 6.08 & $13(50)$ & $31(25)$ & $14(25)$ & $19(200)$ & $12(100)$ \\
\hline $5 g$ & & 6.08 & $10(100)$ & $24(100)$ & $11(50)$ & $11(50)$ & $14(100)$ \\
\hline $5 \mathbf{h}$ & & 6.35 & $11(50)$ & $20(200)$ & $13(100)$ & 09 (100) & $09(25)$ \\
\hline $5 i$ & & 6.96 & $14(200)$ & $37(0.78)$ & $17(100)$ & $13(25)$ & $18(12.5)$ \\
\hline $5 \mathbf{j}$ & & 6.96 & $09(25)$ & $23(100)$ & $15(50)$ & $33(6.25)$ & $07(100)$ \\
\hline $6 a$ & & 6.15 & $12(100)$ & $20(50)$ & $13(12.5)$ & $27(50)$ & $04(100)$ \\
\hline $6 b$ & & 6.71 & $17(50)$ & $24(12.5)$ & $17(25)$ & $24(100)$ & $08(200)$ \\
\hline $6 c$ & & 6.71 & $08(100)$ & $19(50)$ & $07(50)$ & $15(100)$ & $10(10)$ \\
\hline
\end{tabular}




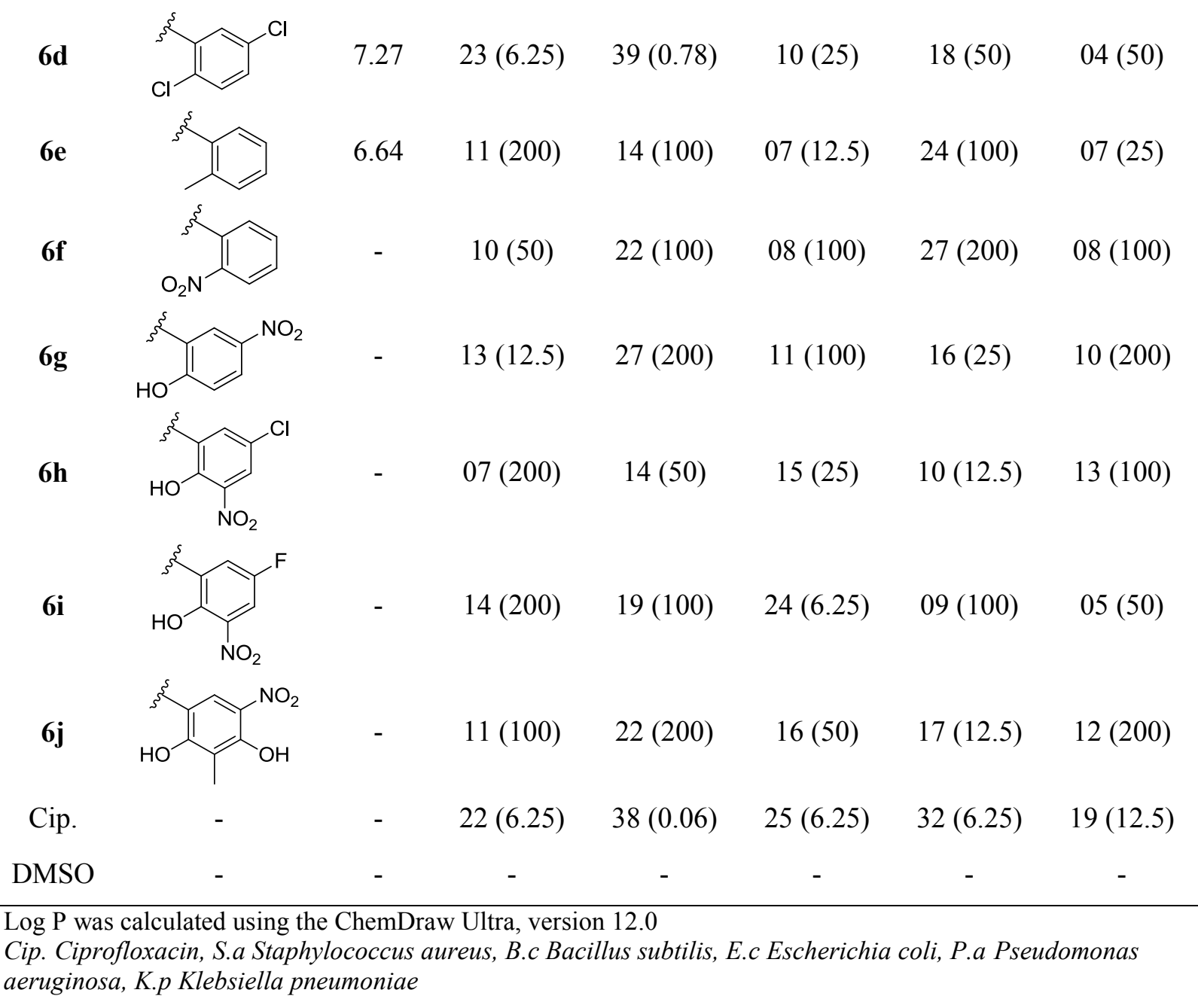

Table 2. In-vitro anti-fungal activity of newly synthesized compounds $\mathbf{5 a - j}$ and $\mathbf{6 a - j}$.

\begin{tabular}{|c|c|c|c|c|c|c|}
\hline \multirow{2}{*}{ Entry } & \multirow{2}{*}{$\mathbf{R} / \mathbf{R}^{\prime}$} & \multirow{2}{*}{$\log P$} & \multicolumn{4}{|c|}{ Zone of Inhibition in $\mathrm{mm}(\mathrm{MIC}$ in $\mu \mathrm{g} / \mathrm{mL})$} \\
\hline & & & A.n & A.f & A.c & C.a \\
\hline $5 \mathbf{a}$ & & 7.03 & $12(12.5)$ & $20(25)$ & $24(100)$ & $21(50)$ \\
\hline $5 \mathbf{b}$ & & 7.03 & $19(25)$ & $11(12.5)$ & $21(100)$ & $13(12.5)$ \\
\hline $5 c$ & & 6.63 & $30(3.12)$ & $15(100)$ & $15(25)$ & $20(50)$ \\
\hline 5d & & - & $11(100)$ & $12(50)$ & $13(200)$ & $23(200)$ \\
\hline
\end{tabular}


$5 e$<smiles>CC(C)c1ccc([N+](=O)[O-])cc1</smiles>

5f<smiles>CC(C)(C)c1cccc(O)c1</smiles>

5g<smiles>CC(C)(C)c1ccc(O)cc1</smiles>

$5 h$<smiles>CC(C)C1CCCCC1</smiles>

$5 i$<smiles>Cc1ccccc1C(C)C</smiles>

$5 j$<smiles>Cc1ccc(C(C)C)cc1</smiles>

6a<smiles>CC(C)c1ccccc1</smiles>

$\mathbf{6 b}$<smiles>CC(C)c1ccccc1Cl</smiles>

6d<smiles>CC(C)c1cc(Cl)ccc1Cl</smiles>

$6 e$<smiles>Cc1ccccc1C(C)C</smiles>

6f<smiles>CC(C)c1ccccc1[N+](=O)[O-]</smiles>

$6 \mathrm{~g}$<smiles>CC(C)c1cc([N+](=O)[O-])ccc1O</smiles>

6i<smiles>CC(C)c1cc(F)cc([N+](=O)[O-])c1O</smiles>

6j<smiles>Cc1c(C(C)C)cc([N+](=O)[O-])c(O)c1O</smiles>

\begin{tabular}{|c|c|c|c|c|}
\hline- & $10(50)$ & $21(12.5)$ & $20(12.5)$ & $17(200)$ \\
\hline 6.08 & $14(100)$ & $19(50)$ & $17(200)$ & $11(100)$ \\
\hline 6.08 & $18(50)$ & $11(50)$ & $24(50)$ & $13(25)$ \\
\hline 6.35 & $20(200)$ & $17(100)$ & $16(50)$ & $15(50)$ \\
\hline 6.96 & $17(25)$ & $13(6.25)$ & $22(100)$ & $17(100)$ \\
\hline 6.96 & $24(12.5)$ & $27(0.78)$ & $20(12.5)$ & $22(25)$ \\
\hline 6.15 & $21(100)$ & $15(25)$ & $17(100)$ & $15(100)$ \\
\hline 6.71 & $15(100)$ & $12(50)$ & $08(50)$ & $17(12.5)$ \\
\hline 6.71 & $11(25)$ & $14(100)$ & $06(200)$ & $13(100)$ \\
\hline 7.27 & $31(3.12)$ & $10(100)$ & $17(50)$ & $35(0.78)$ \\
\hline 6.64 & 09 (200) & $07(50)$ & $15(100)$ & $24(12.5)$ \\
\hline - & $17(200)$ & $08(100)$ & $13(200)$ & $23(12.5)$ \\
\hline- & $16(50)$ & $13(50)$ & $14(12.5)$ & $17(200)$ \\
\hline 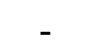 & $09(25)$ & $28(0.78)$ & $18(25)$ & $16(25)$ \\
\hline
\end{tabular}

$18(12.5) \quad 10(12.5) \quad 32(0.78) \quad 22(100)$

$14(6.25) \quad 21(6.25) \quad 24(100) \quad 20(50)$ 
Ket.

$30(\leq 3)$

$29(\leq 1)$

$31(\leq 1)$

$33(\leq 1)$

\section{DMSO}

Log P was calculated using the ChemDraw Ultra, version 12.0

Ket. Ketoconazole, A.n Aspergillus niger, A.f Aspergillus fumigates, A.c Aspergillus clavatus, C.a Candida albicans

Table 3. In-vitro anti-tuberculosis activity of newly synthesized compounds $\mathbf{7 a - j}$ and $\mathbf{9 a - j}$.

\begin{tabular}{|c|c|c|c|c|}
\hline \multirow{2}{*}{ Entry } & \multirow{2}{*}{$\mathbf{R} / \mathbf{R}^{\prime}$} & \multirow{2}{*}{$\log P$} & \multicolumn{2}{|c|}{ L-J MIC method ${ }^{\mathrm{a}}$} \\
\hline & & & $\operatorname{MIC}(\mu \mathrm{g} / \mathrm{mL})$ & $\%$ Inhibition \\
\hline $5 \mathbf{a}$ & & 7.03 & 3.12 & 99 \\
\hline $5 b$ & & 7.03 & 12.5 & 95 \\
\hline $5 c$ & & 6.63 & 100 & 89 \\
\hline 5d & & - & 100 & 88 \\
\hline $5 e$ & & - & 200 & 80 \\
\hline $5 f$ & & 6.08 & 50 & 92 \\
\hline $5 \mathrm{~g}$ & & 6.08 & 50 & 91 \\
\hline $5 \mathrm{~h}$ & & 6.35 & 12.5 & 96 \\
\hline $5 i$ & & 6.96 & 25 & 92 \\
\hline $5 \mathbf{j}$ & & 6.96 & 100 & 88 \\
\hline $6 a$ & & 6.15 & 100 & 89 \\
\hline
\end{tabular}


6b<smiles>CC(C)c1ccccc1Cl</smiles>

6c<smiles>[3H]C([3H])c1ccc(Cl)cc1</smiles>

6d

$6 e$

6f<smiles>CC(C)c1cc(Cl)ccc1Cl</smiles>

6.64<smiles>Cc1ccccc1C(C)C</smiles><smiles>CC(C)c1ccccc1[N+](=O)[O-]</smiles>

$6 \mathrm{~g}$

6h<smiles>CC(C)(C)c1cc([N+](=O)[O-])ccc1O</smiles><smiles>Cc1c(O)c([N+](=O)[O-])cc(C(C)(C)C)c1O</smiles>

50

90

93

99

90

89

93

95<smiles>CC(C)c1cc(F)cc([N+](=O)[O-])c1O</smiles>

$\begin{array}{ll}- & 3.12\end{array}$

99

6j

93




\begin{tabular}{|c|c|c|c|c|c|c|}
\hline $5 c$ & $237-239$ & 77 & $\mathrm{C}_{26} \mathrm{H}_{20} \mathrm{FN}_{7} \mathrm{O}$ & $\begin{array}{c}66.94 \\
(67.09)\end{array}$ & $\begin{array}{c}4.34 \\
(4.33)\end{array}$ & $\begin{array}{c}21.04 \\
(21.06)\end{array}$ \\
\hline $5 d$ & $269-271$ & 76 & $\mathrm{C}_{26} \mathrm{H}_{20} \mathrm{~N}_{8} \mathrm{O}_{3}$ & $\begin{array}{c}63.58 \\
(63.41)\end{array}$ & $\begin{array}{c}4.08 \\
(4.09)\end{array}$ & $\begin{array}{c}22.70 \\
(22.75)\end{array}$ \\
\hline $5 e$ & $255-257$ & 81 & $\mathrm{C} 26 \mathrm{H} 20 \mathrm{~N} 8 \mathrm{O} 3$ & $\begin{array}{c}63.32 \\
(63.41)\end{array}$ & $\begin{array}{c}4.10 \\
(4.09)\end{array}$ & $\begin{array}{c}22.81 \\
(22.75)\end{array}$ \\
\hline $5 f$ & $176-178$ & 74 & $\mathrm{C}_{26} \mathrm{H}_{21} \mathrm{~N}_{7} \mathrm{O}_{2}$ & $\begin{array}{c}67.52 \\
(67.38)\end{array}$ & $\begin{array}{c}4.56 \\
(4.57)\end{array}$ & $\begin{array}{c}21.11 \\
(21.15)\end{array}$ \\
\hline $5 g$ & $188-190$ & 70 & $\mathrm{C}_{26} \mathrm{H}_{21} \mathrm{~N}_{7} \mathrm{O}_{2}$ & $\begin{array}{c}67.19 \\
(67.38)\end{array}$ & $\begin{array}{c}4.56 \\
(4.57)\end{array}$ & $\begin{array}{c}21.10 \\
(21.15)\end{array}$ \\
\hline $5 \mathbf{h}$ & $117-119$ & 68 & $\mathrm{C}_{26} \mathrm{H}_{27} \mathrm{~N}_{7} \mathrm{O}$ & $\begin{array}{c}68.96 \\
(68.85)\end{array}$ & $\begin{array}{c}5.99 \\
(6.00)\end{array}$ & $\begin{array}{c}21.56 \\
(21.62)\end{array}$ \\
\hline $5 \mathbf{i}$ & $247-249$ & 65 & $\mathrm{C}_{27} \mathrm{H}_{23} \mathrm{~N}_{7} \mathrm{O}$ & $\begin{array}{c}70.10 \\
(70.27)\end{array}$ & $\begin{array}{c}5.01 \\
(5.02)\end{array}$ & $\begin{array}{c}21.18 \\
(21.24)\end{array}$ \\
\hline $5 \mathbf{j}$ & $222-224$ & 73 & $\mathrm{C}_{27} \mathrm{H}_{23} \mathrm{~N}_{7} \mathrm{O}$ & $\begin{array}{c}70.09 \\
(70.27)\end{array}$ & $\begin{array}{c}5.03 \\
(5.02)\end{array}$ & $\begin{array}{c}21.27 \\
(21.24)\end{array}$ \\
\hline $6 \mathbf{a}$ & $167-169$ & 69 & $\mathrm{C}_{28} \mathrm{H}_{22} \mathrm{~N}_{6} \mathrm{O}_{2}$ & $\begin{array}{c}70.80 \\
(70.87)\end{array}$ & $\begin{array}{c}4.68 \\
(4.67)\end{array}$ & $\begin{array}{c}17.67 \\
(17.71)\end{array}$ \\
\hline $6 b$ & $131-133$ & 70 & $\mathrm{C}_{28} \mathrm{H}_{21} \mathrm{ClN}_{6} \mathrm{O}_{2}$ & $\begin{array}{c}65.93 \\
(66.08)\end{array}$ & $\begin{array}{c}4.15 \\
(4.16)\end{array}$ & $\begin{array}{c}16.55 \\
(16.51)\end{array}$ \\
\hline $6 c$ & $141-143$ & 77 & $\mathrm{C}_{28} \mathrm{H}_{21} \mathrm{ClN}_{6} \mathrm{O}_{2}$ & $\begin{array}{c}66.26 \\
(66.08)\end{array}$ & $\begin{array}{c}4.17 \\
(4.16)\end{array}$ & $\begin{array}{c}16.47 \\
(16.51)\end{array}$ \\
\hline 6d & 184-186 & 72 & $\mathrm{C}_{28} \mathrm{H}_{20} \mathrm{C}_{12} \mathrm{~N}_{6} \mathrm{O}_{2}$ & $\begin{array}{c}61.78 \\
(61.89)\end{array}$ & $\begin{array}{c}3.70 \\
(3.71)\end{array}$ & $\begin{array}{c}15.45 \\
(15.47)\end{array}$ \\
\hline $6 e$ & $205-207$ & 67 & $\mathrm{C}_{29} \mathrm{H}_{24} \mathrm{~N}_{6} \mathrm{O}_{2}$ & $\begin{array}{c}71.11 \\
(71.30)\end{array}$ & $\begin{array}{c}4.94 \\
(4.95)\end{array}$ & $\begin{array}{c}17.16 \\
(17.20)\end{array}$ \\
\hline $6 f$ & $228-230$ & 73 & $\mathrm{C}_{28} \mathrm{H}_{21} \mathrm{~N}_{7} \mathrm{O}_{4}$ & $\begin{array}{c}64.55 \\
(64.73)\end{array}$ & $\begin{array}{c}4.07 \\
(4.07)\end{array}$ & $\begin{array}{c}18.82 \\
(18.87)\end{array}$ \\
\hline $6 \mathrm{~g}$ & $178-180$ & 70 & $\mathrm{C}_{28} \mathrm{H}_{21} \mathrm{~N}_{7} \mathrm{O}_{5}$ & $\begin{array}{l}62.88 \\
(62.8)\end{array}$ & $\begin{array}{c}3.94 \\
(3.95)\end{array}$ & $\begin{array}{c}18.33 \\
(18.31)\end{array}$ \\
\hline $6 h$ & $150-152$ & 69 & $\mathrm{C}_{28} \mathrm{H}_{20} \mathrm{ClN}_{7} \mathrm{O}_{5}$ & $\begin{array}{c}58.87 \\
(59.00)\end{array}$ & $\begin{array}{c}3.55 \\
(3.54)\end{array}$ & $\begin{array}{l}17.16 \\
(17.2)\end{array}$ \\
\hline $6 \mathbf{i}$ & 189-191 & 74 & $\mathrm{C}_{28} \mathrm{H}_{20} \mathrm{FN}_{7} \mathrm{O}_{5}$ & $\begin{array}{c}60.94 \\
(60.76)\end{array}$ & $\begin{array}{c}3.63 \\
(3.64)\end{array}$ & $\begin{array}{c}17.66 \\
(17.71)\end{array}$ \\
\hline $\mathbf{6 j}$ & 197-199 & 72 & $\mathrm{C}_{29} \mathrm{H}_{23} \mathrm{~N}_{7} \mathrm{O}_{6}$ & $\begin{array}{c}61.50 \\
(61.59)\end{array}$ & $\begin{array}{c}4.09 \\
(4.10)\end{array}$ & $\begin{array}{c}17.37 \\
(17.34)\end{array}$ \\
\hline
\end{tabular}

\section{BIOLOGICAL EVALUATION}

All newly synthesized $s$-triazinyl schiff bases and chalcone derivatives were accessed for their in vitro antimicrobial evaluation against five bacterial strains (Staphylococcus aureus MTCC 96, Bacillus subtilis MTCC 441, Escherichia coli MTCC 739, Pseudomonas aeruginosa MTCC 741 and Klebsiella pneumoniae MTCC 109) and four fungal strains (Aspergillus niger MTCC 282, Aspergillus fumigates MTCC 343, Aspergillus clavatus MTCC 1323, Candida albicans MTCC 183) using broth dilution technique. 
Ciprofloxacin and ketoconazole were used as standard control drugs for antibacterial and antifungal activity, respectively. Further, all the newly synthesized derivatives were evaluated for their in vitro antimycobacterial activity (against Mycobacterium tuberculosis H37Rv) by Lowenstein-Jensen MIC method using Isoniazid, Rifampicin, Ethambutol, and Pyrazinamide as standard control drugs.

\section{1. In vitro antibacterial activity}

The analysis of antibacterial screening data (Table 1) reveals that some of the scaffolds were found to exhibit good to moderate efficacy against particular bacterial strain. Among them, the halogen group containing moieties $\mathbf{5 a}$ and $\mathbf{5 c}$ were found active against $S$. aureus MTCC 96 and E. coli MTCC 739, respectively, both at MIC $6.25 \mu \mathrm{g} / \mathrm{mL}$. The electron donating methyl group possessing compound $\mathbf{5 i}$ exhibited superior inhibition profile against B. subtilis MTCC 441 and $K$. pneumoniae MTCC 109 at MIC $0.78 \mu \mathrm{g} / \mathrm{mL}$ and MIC 12.5 $\mu \mathrm{g} / \mathrm{mL}$ respectively. Again, the methyl group containing motif $\mathbf{5 j}$ showed activity against $P$. aeruginosa MTCC 741 at MIC $6.25 \mu \mathrm{g} / \mathrm{mL}$. The chalcone derivative having 2,5-dichloro phenyl residue 6d was found to exhibit higher potential against $S$. aureus MTCC 96 and $B$. subtilis MTCC 441 at MIC $6.25 \mu \mathrm{g} / \mathrm{mL}$ and $0.78 \mu \mathrm{g} / \mathrm{mL}$, respectively. The final chalcone compound 6i gave significant inhibition against E. coli MTCC 739 at MIC $6.25 \mu \mathrm{g} / \mathrm{mL}$.

\section{2. In vitro antifungal activity}

In vitro antifungal activity (Table 2) pointed out that the final schiff base and chalcone derivatives bearing halogen group proved to be highly potent against the specific fungal strain. The final schiff base moiety $\mathbf{5 c}$ having fluoro group showed inhibitory effect on $A$. niger MTCC 282 at MIC $3.12 \mu \mathrm{g} / \mathrm{mL}$. The methyl group containing schiff base $\mathbf{5} \mathbf{j}$ were found highly potent against $A$. fumigates MTCC 343 at MIC $0.78 \mu \mathrm{g} / \mathrm{mL}$. The triazinyl chalcone derivatives endowed with 2,5-dichloro phenyl residue was found highly active against $A$. niger MTCC 282 and C. albicans MTCC 183 at MIC $3.12 \mu \mathrm{g} / \mathrm{mL}$ and MIC $0.78 \mu \mathrm{g} / \mathrm{mL}$, respectively. The compounds $6 \mathrm{~h}$ and $6 \mathrm{i}$ possessing chloro and fluoro group respectively, were found to possess superior inhibition profile against A. fumigates MTCC 343, A. clavatus MTCC 1323, respectively, both at MIC $0.78 \mu \mathrm{g} / \mathrm{mL}$.

\section{3. In vitro antituberculosis activity}

In vitro antituberculosis activity (Table 3 ) was performed for the all novel synthesized s-triazinyl schiff bases and chalcone derivatives against the tubercular strain $M$. tuberculosis H37Rv. All the novel congeners were evaluated for anti-TB activity using LowensteinJensen MIC method. Among the all scaffolds, the chloro group possessing compounds $\mathbf{5 a}$ and 6d showed highest inhibition for tubercular strain at MIC $3.12 \mu \mathrm{g} / \mathrm{mL}$. The fluoro group containing derivative 6i also exhibited very good inhibitory effect on the mentioned tubercular strain at MIC $3.12 \mu \mathrm{g} / \mathrm{mL}$.

\section{CONCLUSION}

The current study mainly focused on the development of novel $s$-triazinyl schiff base and chalcone derivatives in good yield and with a huge range of their pharmaceutical applications. It can be concluded from the above results that many of the synthesized derivatives exhibit good to moderate activity against specific microbial strain. Out of the all 
synthesized scaffolds, the final analogues with halogen/methyl group i.e., $\mathbf{5 a}, \mathbf{5 c}, \mathbf{5 i}, \mathbf{5 j}, \mathbf{6 d}, \mathbf{6 i}$ and $\mathbf{6 h}$ were proved to be highly efficient against specific bacterial, fungal and $M$. tuberculosis H37Rv strain as well.

\section{Acknowledgements}

Authors are very thankful to Prof. Nisha K. Shah, Head, Department of Chemistry, School of Sciences, Gujarat University, Ahmedabad, India for her kind cooperation for providing support and research facility. The authors wish to offer their deep gratitude to TB-care Laboratory, Ahmedabad, India for carrying out the biological screenings. We are also thankful to NFDD-Rajkot, Gujarat, India for carrying out IR , ${ }^{1} \mathrm{H}$ NMR and ${ }^{13} \mathrm{C}$ NMR analysis.

\section{References}

[1] World Health Organization. http://www.who.int/mediacentre/factsheets/fs194/en/ (accessed July 17, 2014).

[2] Centers for Disease Control and Prevention (CDC); Fact Sheet. http://www.cdc.gov/tb/publications/factsheets/drtb/mdrtb.htm (accessed July 17, 2014).

[3] World Health Organization; Fact sheet $\mathrm{N}^{\circ} 104$. http://www.who.int/mediacentre/factsheets/fs104/en/ (accessed July 17, 2014).

[4] D. R. Shah, H. P. Lakum, K. H. Chikhalia, International Letters of Chemistry, Physics and Astronomy 17(2) (2014) 207-219.

[5] H. P. Lakum, D. V. Desai, K. H. Chikhalia, Heterocyclic Communications 19 (2013) 351-355.

[6] R. V. Patel, P. Kumari, D. P. Rajani, K. H. Chikhalia, European journal of medicinal chemistry 46 (2011) 4354-4365.

[7] R. Menicagli, S. Samaritani, G. Signore, F. Vaglini, L. Dalla Via, Journal of Medicinal Chemistry 47 (2004) 4649-4652.

[8] K. Arya, A. Dandia, Bioorganic \& medicinal chemistry letters 17 (2007) 3298-3304.

[9] A. Kumar, K. Srivastava, S. Raja Kumar, S. K. Puri, P. M. Chauhan, Bioorganic \& medicinal chemistry letters 19 (2009) 6996-6999.

[10] X. Chen, P. Zhan, C. Pannecouque, J. Balzarini, E. De Clercq, X. Liu, European journal of medicinal chemistry 51 (2012) 60-66.

[11] K. Srinivas, U. Srinivas, V. J. Rao, K. Bhanuprakash, K. H. Kishore, U. S. N. Murty, Bioorganic \& medicinal chemistry letters 15 (2005) 1121-1123.

[12] U. P. Singh, R. K. Singh, H. R. Bhat, Y. P. Subhashchandra, V. Kumar, M. K. Kumawat, P. Gahtori, Medicinal Chemistry Research 20 (2011) 1603-1610.

[13] S. H. Abdel-Hafez, Phosphorus, Sulfur and Silicon 178 (2003) 2563-2579.

[14] V. R. Avupati, R. P. Yejella, V. R. Parala, K. N. Killari, V. M. R. Papasani, P. Cheepurupalli, V. R. Gavalapu, B. Boddeda, Bioorganic \& medicinal chemistry letters 23 (2013) 5968-5970. 
[15] A. Solankee, R. Patel, K. Patel, Chim. Sin 3 (2011) 317-324.

[16] S. John Joseph, R. Arulkumaran, D. Kamalakkannan, S. P. Sakthinathan, R. Sundararajan, R. Suresh, S. Vijayakumar, K. Ranganathan, N. Kalyanasundaram, G. Vanangamudi, G. Thirunarayanan, International Letters of Chemistry, Physics and Astronomy 4 (2014) 48-65.

[17] S. John Joseph, R. Arulkumaran, D. Kamalakkannan, S. P. Sakthinathan, R. Sundararajan, R. Suresh, S. Vijayakumar, K. Ranganathan, N. Kalyanasundaram, G. Vanangamudi, G. Thirunarayanan, International Letters of Chemistry, Physics and Astronomy 4 (2014) 48-65.

[18] S. John Joseph, D. Kamalakkannan, R. Arulkumaran, S. P. Sakthinathan, R. Suresh, R. Sundararajan, S. Vijayakumar, K. Ranganathan, G. Vanangamudi, G. Thirunarayanan, International Letters of Chemistry, Physics and Astronomy 5 (2014) 99-123.

[19] Nirali S. Mewada, Dhruvin R. Shah, Kishor H. Chikhalia, International Letters of Chemistry, Physics and Astronomy 17(3) (2014) 281-294.

[20] S. Vijayakumar, R. Arulkumaran, R. Sundararajan, S. P. Sakthinathan, R. Suresh, D. Kamalakkannan, K. Ranganathan, K. Sathiyamoorthy, V. Mala, G. Vanangamudi, G. Thirunarayanan, International Letters of Chemistry, Physics and Astronomy 9(1) (2013) 68-86. 